\title{
ARSH-FATI a Novel Meta-heuristic for Cluster Head Selection in Wireless Sensor Networks
}

\author{
Haider Ali, Umair Ullah Tariq, Mubashir Hussain, Liu Lu, Member, IEEE, John Panneerselvam, and \\ Xiaojun Zhai
}

\begin{abstract}
Wireless Sensor Network (WSN) consists of a large number of sensor nodes distributed over a certain target area. The WSN plays a vital role in surveillance, advanced healthcare, and commercialized industrial automation. Enhancing energyefficiency of the WSN is a prime concern because higher energy consumption restricts the Lifetime (LT) of the network. Clustering is a powerful technique widely adopted to increase LT of the network and reduce the transmission energy consumption. In this paper we develop a novel ARSH-FATI based Cluster Head Selection (ARSH-FATI-CHS) algorithm integrated with a heuristic called Novel Ranked based Clustering (NRC) to reduce the communication energy consumption of the sensor nodes while efficiently enhancing LT of the network. Unlike other population based algorithms ARSH-FATI-CHS dynamically switches between exploration and exploitation of the search process during run-time to achieve higher performance trade-off and significantly increase LT of the network. ARSH-FATI-CHS considers the residual energy, communication distance parameters, and workload during Cluster Heads (CHs) selection. We simulate our proposed ARSH-FATI-CHS and generate various results to determine the performance of the WSN in terms of LT. We compare our results with state-of-the-art Particle Swarm Optimization (PSO) and prove that ARSH-FATI-CHS approach improves the LT of the network by $\sim 25 \%$.
\end{abstract}

Index Terms-ARSH-FATI, Wireless Sensor Network, Sensor Nodes, Cluster Head, Clustering, Lifetime.

\section{INTRODUCTION}

I NTERNET-of-Things (IoT) is a $21^{\text {st }}$ century technological revolution and its potential applications have already exceeded the expectations. According to Cisco, it is expected that there will be 50 billion devices connected to the Internet by 2020 [1], [2]. The basic concept of this idea is the pervasive presence of a variety of objects or things around us. In other words IoT paradigm interconnects computers, machines, people, actuators, and digital devices on a large-scale for applications including surveillance, advanced healthcare, smart agriculture, industrial automation, industrial manufacturing, and intelligent transportation [3], [4]. Unquestionably IoT has its impact on several aspects of the everyday life and

Haider Ali and John Panneerselvam are with the Department of Electronics, Computing and Mathematics, University of Derby, Derby, United Kingdom e-mail: h.ali9@unimail.derby.ac.uk.

Umair Ullah Tariq is with the School of Engineering and Technology, Central Queensland University, Sydney, Australia e-mail: u.tariq@cqu.edu.au.

Mubashir Hussain is with the School of Computer Science and Engineering, University of New South Wales, Sydney, NSW, Australia e-mail: mubashir@live.com.

Liu Lu is with the School of Informatics, University of Leicester, Leicester, United Kingdom e-mail: 1.liu@leicester.ac.uk.

Xiaojun Zhai is with the School of Computer Science and Electronic Engineering at the University of Essex, Colchester, UK. e-mail:xzhai@essex.ac.uk. behavior of the users [4], [5]. Wireless Sensor Network (WSN) is an integrated part of the IoT and provides the collected information to the cloud about the physical world collected by dedicated digital edge devices called sensor nodes [6], [7], [8], [9]. WSN is responsible for efficiently gathering the information in several IoT based applications for example in traffic monitoring wireless senors nodes are embedded on intersections and roadways to collect the traffic data for traffic signal adjustment, avoiding congestion, and and road accident severity. In the video streaming the multimedia data content is streamed over the network in an encoded form while the video is displayed to the end user and/or professional either in a recorded or pre-recorded manner [10].

The design of sustainable WSNs is a challenging issue and the energy-constrained sensors nodes are expected to efficiently run autonomously for a long period of time. However, it is cost-prohibitive to replace the batteries with no residual energy often it is impossible in the hostile environments [7], [11], [12]. Thus, one of the major constraints in WSNs is that sensor nodes have limited power source and replacement of the battery is mostly impossible. Energy-efficiency in IoT has attracted a lot of attention from the researchers over the last few years, paving the way for initiating an area called green IoT. The energy consumption can be reduced at different aspects of IoT such as applications, services, and communications where energy-efficient utilization is needed to enable a green IoT environment [13]. Therefore, energy consumption reduction of the sensor nodes is challenging facet in the development of large scale WSNs to reduce the transmission energy consumption [14], [15]. A sensor node in WSNs is typically consisted of four fundamental components demonstrated in Fig. (1). Each component of the sensor node is briefly explained as follows [16]:

1) Sensing Unit (SU): It is used for acquiring analogue data from a certain Field-of-View (FoV). The information detected in analogue form is converted into digital format by using Analogue-to-Digital (ADC) converts.

2) Processing Unit (PU): It intelligently process the collected data from the target area through electromechanical sensors. It is a microprocessor and/or microcontroller with integrated memory.

3) Wireless Communication Unit (WCU): It is deployed for communicating the data and/or information commonly. It is a short-range transceiver system commonly based on standards such as IEEE 802.14.3, IEEE 802.15.4 or $\mathrm{ZigBee}^{\mathrm{TM}}$. 
4) Power Source: It is mostly a DC battery with limited residual energy. Regulated supply for the data collection, processing, and transmission subsystems.

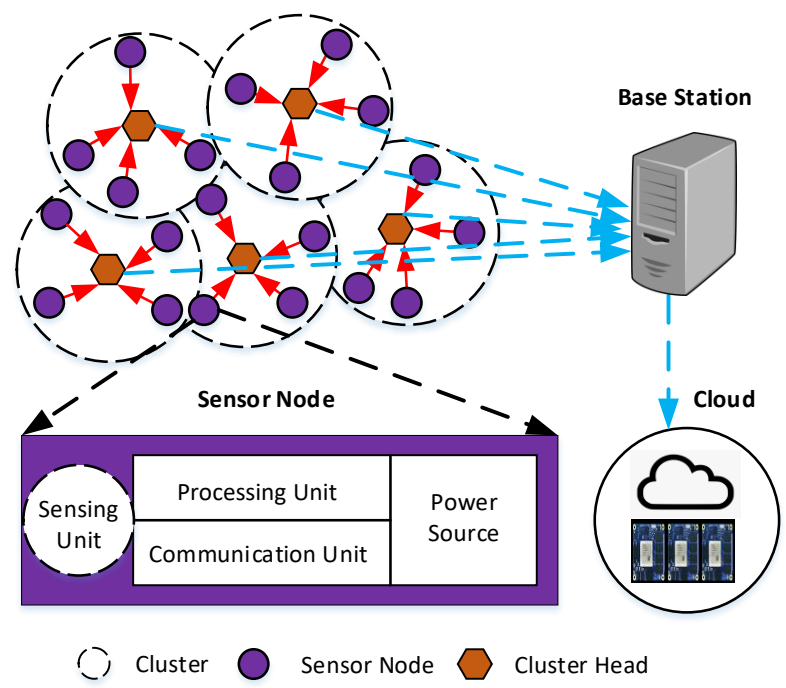

Fig. (1) Wireless sensor network architecture

The communication unit of the sensor node consumes higher energy compared to the sensing and processing units [17], [16]. Therefore, sensor nodes clustering is an effective technique deployed to increase the energy-efficiency of the WSN. In clustering process, the non-cluster nodes basically join a neighboring Cluster Head $(\mathrm{CH})$ to form a cluster. This process of cluster formation plays a significant role to decrease the energy consumption of a network [12]. Clustering in WSN not only provides data aggregation, scalability, bandwidth conservation but also prolongs LT of the network by decreasing the communication energy consumption of the sensor nodes. In clustering process, the sensor nodes are partitioned into groups called clusters. Where each cluster has its own leader known as $\mathrm{CH}$. The $\mathrm{CH}$ collects the data within the cluster from its member sensor nodes, aggregates the collected data and transmits it to the Base Station (BS). Data collected by the $\mathrm{CH}$ is either transmitted directly to the BS or through intermediate $\mathrm{CHs}$ and/or sensor nodes i.e. using multi hop communication. Information from the $\mathrm{BS}$ is transmitted to the Cloud for further processing and visualization purposes as demonstrated in Fig. 1 [14], [12]. The $\mathrm{CH}$ selection is a well known NP-hard problem [18]. Subsequently, various searching based heuristics have been developed using Genetic Algorithm (GA) [19], Ant Colony Optimization (ACO) [20], Particle Swarm Optimization (PSO) [18], Differential Evolution (DE) [21], and Simulated Annealing (SA) [22]. Among these algorithms, PSO is widely used for cluster formation to enhance LT of the network.

\section{A. Contributions and Innovations}

In this section we present our innovations and contributions. First we define our problem given as follows:

Problem Statement: Performing an energy-efficient sensor nodes clustering to reduce transmission energy consumption and increase the overall LT of a network using evolutionary algorithm by considering various parameters in the fitness fiction. Concisely, in this paper we investigate to reduce the transmission energy consumption of the sensor nodes in WSN and present ARSH-FATI [23] based Cluster Head Selection (ARSH-FATI-CHS) meta-heuristic integrated with Ranked based Clustering (NRC) heuristic. The major innovations and contributions of this paper are given as follows:

1) We develop a novel meta-heuristic, ARSH-FATI-CHS that dynamically switches between exploration and exploitation search modes during run-time of the $\mathrm{CHs}$ selection process for better performance trade-off.

2) We present an algorithm NRC that estimate the LT of the sensor nodes and guides the ARSH-FATI-CHS to a better and energy-efficient clustering to enhance the LT of the network.

3) We propose a fitness function that considers various parameters such as residual energy, communications distance of the sensor nodes, and workload on the selected CHs.

4) We compare our clustering scheme, ARSH-FATI-CHS with state-of-the-art PSO based approach presented by Rao et al. [14] and it achieves an overall LT improvement of $\sim 25 \%$ when simulated for various number of sensor nodes. Similarly, ARSH-FATI-CHS also outperforms LEACH [24] and PSO-C [18] while attains an average LT improvement of $\sim 40 \%$ and $\sim 60 \%$ respectively.

We organize the rest of the paper as follows; Section II discusses the related work performed so far. Section III presents the preliminaries. Section IV explains our novel $\mathrm{CHs}$ selection and clustering algorithms. Experimental results and discussions are presented in Section $\mathrm{V}$ followed by conclusion of this paper in Section VI.

\section{RELATED WORK}

Motivated by the fact that energy-efficiency plays a vital role in the WSN to enhance LT of the network, different algorithms for energy-aware sensor nodes clustering have been investigated in the literature.

One of the most popular and widely used sensor nodes clustering algorithm is Low-Energy Adaptive Clustering Hierarchy (LEACH) [24]. LEACH is a probabilistic approach that randomly selects $\mathrm{CH}$ in each round. Though, LEACH attains a significant energy consumption reduction while prolonging LT of the network compared to Static Clustering and Minimum Transmission Energy (MTE) algorithm however, some disadvantages are associated with it. For example, LEACH can select a SN with least residual energy as a CH subsequently, which adversely affects the network's LT. Numerous other clustering algorithms have been developed by the researchers to improve the efficiency of LEACH. For instance Power-Efficient GAthering in Sensor Information Systems (PEGASIS) [25] that is a chain based approach. PEGASIS organizes SNs into a chain while each SN communicates only with neighbour SNs. Each SN in the chain takes turns in every round to transmit data to the base station. PEGASIS saves more energy when compared to LEACH 
but it is not suitable for large-sized networks due to its instability and high delays. Loscri et al. [26] presented a twolevel hierarchical approach called TL-LEACH. TL-LEACH efficiently distributes the energy load among the SNs for large-sized networks. It significantly enhances LT compared to LEACH but causes an extra overhead for selecting secondary CHs. Xiangning et al. [27] improved LEACH by considering the residual energy of the SNs during $\mathrm{CH}$ selection process and developed a protocol called Energy-LEACH (E-LEACH). However, this protocols only minimizes the average distance between $\mathrm{CHs}$ and the non- $\mathrm{CH}$ nodes while fails to consider the distance between the BS and CHs. Yassein et al. [28] developed a Voice-LEACH (V-LEACH) protocol. V-LEACH also selects a voice- $\mathrm{CH}$ besides a $\mathrm{CH}$ in the cluster. The voice$\mathrm{CH}$ acts as a $\mathrm{CH}$ when there is no residual energy left in the $\mathrm{CH}$. Though, V-LEACH performs better than LEACH in terms of energy-efficiency however, extra amount of energy is utilized for selecting the voice-CHs during clustering phase. Al-Baz et al. [29] improved the performance of LEACH and developed an algorithm called Node Ranked-LEACH (NRLEACH). The NR-LEACH selects $\mathrm{CH}$ by considering cost and number of links between the sensor nodes. This enhances LT of the network and distributes energy load among the sensor nodes. However, this approach is not suitable for large-scale networks. LEACH-C [30], LEACH-GA [31], LEACH-C [32], and DE-LEACH [33] integrated evolutionary algorithms with LEACH to improve its performance. Recently, Lin et al. [34] took an advantage of a game model to select $\mathrm{CHs}$ and proposed a routing protocol called Game theory based Energy Efficient Clustering routing protocol (GEEC). It adopts an evolutionary game theory mechanism for achieving energy balance and longevity. Wang et al. [35] developed an Energy-Efficient Compressive Sensing-based clustering Routing (EECSR) protocol and combined merits of the clustering strategy and compressive sensing based scheme.

In the analytical study presented by zungeru et al. [36], it has been discussed that evolutionary meta-heuristic algorithms based sensor nodes clustering offers a significant energy savings. Since finding m-optimal clusters is NP-hard problem [37] therefore, in the last decade numerous search based metaheuristics have been proposed in the literature for efficient $\mathrm{CHs}$ selection. Subsequently, researchers developed meta-heuristic based algorithms for efficient cluster formation in WSN to enhance LT of the network. Centralized LEACH (LEACHC) [32] is among the earliest meta-heuristic based clustering approach where SA is used for solving the clustering problem. In LEACH-C, initially all sensor nodes transmit their location and energy information to the BS where SA is applied to determine the clusters. In terms of energy savings LEACHC outperforms LEACH [24]. Guru et al. [38] proposed a cluster formation scheme using PSO using a fitness function that includes the sink distance and intra-cluster distance. This approach fail to consider the residual energy of the SNs. Latiff et al. presented an algorithm called PSO-C for energy-efficient clustering in WSN. PSO-C considers an average intra-cluster distance and total initial energy of all SNs to the total current energy of all CHs. However, PSO-C assigns non-CH nodes in the cluster formation to the nearest $\mathrm{CH}$ which can potentially decrease the energy-efficiency of the network. Singh et al. [39] presented a PSO based energy-efficient $\mathrm{CH}$ selection algorithm called PSO-Semi Distributed (PSO-SD) and used a fitness function that considering residual energy, distance, and node density. The disadvantage of PSO-SD fail to consider cluster formation phase which consequently can reduce the energy efficiency of the network. Rao et al. developed energyaware clustering approach using novel Chemical Reaction Optimization (nCRO) heuristic. The nCRO prolongs LT of the network but the main drawback is that the selected CHs directly communicating to the $\mathrm{BS}$, which is not desirable for large scale network [40]. Kuila and Jana [21] used a novel $\mathrm{DE}$ algorithm for SNs clustering to enhance energy-savings. Though, this scheme efficiently prolongs SNs LT but does not consider sink distance in the process of clustering moreover, $\mathrm{CHs}$ are selected randomly. Kuila et al. [41] developed a load balancing algorithm using DE for better energy-savings. The demerit of this approach includes selecting the $\mathrm{CHs}$ randomly while not considering energy and distance parameters. Gupta and Jana [19] presented a GA based clustering approach while considering the residual energy of the SNs and the distance from their corresponding CHs. Zhang et al. [22] clustered the SNs using $\mathrm{SA}$ and GA while $\mathrm{CH}$ s are selected by estimating the average energy of the cluster. $\mathrm{A} \mathrm{CH}$ is selected if a $\mathrm{SN}$ has higher residual energy than the average energy of the cluster. Hoang et al. [42] developed Harmony search algorithm based clustering protocol (HSACP) to decrease the intra-cluster distances between the cluster members and their $\mathrm{CHs}$. However energy-efficiency can decrease if the workload on CHs is not considered. Rao et al. [14] developed a PSO based energy-efficient $\mathrm{CH}$ selection (PSO-ECHS) algorithm to increase LT of the network. The fitness function in PSOECHS considers parameters including sink distance, intracluster distance, and residual energy of the SNs. The clustering algorithms in [41], [19], [22], [14] increased the LT of the network but there are numerous number of parameters involved required to tune these heuristics for achieving a balance between exploitative and exploitative search modes to attain higher energy-efficiency. Cisse et al. [43] developed an Energy Aware Neighbor Oriented Clustering (EANOC) to select the $\mathrm{CH}$ on the basis of Received Signal Strength Indicator (RSSI) value. However, sensor nodes consume energy due to propagating RSSI in multi-directions which consequently affects the overall energy-efficiency of the network.

There is an abundant literature on energy savings for WSNs and various methods and/or algorithms have been presented in the last few years but there is still much ongoing research to optimize the energy consumption of the battery-limited wireless networks. Our proposed $\mathrm{CHs}$ selection integrated with clustering technique using a novel meta-heuristic for enhancing LT of the network possesses the following advantages over other existing approaches:

1) Unlike, these sensor nodes clustering algorithms in [24], [25], [27], [18], [22], [42], we use a fitness function that considers the residual energy, distance parameters, and workload on the selected CHs to prolong the overall LT of the network. 
2) State-of-the-art searching based algorithms in [41], [19], [22], [14] are complex and require different parameters tuning to attain energy-efficient solution. Our novel meta-heuristic ARSH-FATI-CHS dynamically switches between exploitative and exploitative modes at run-time for better and efficient performance trade-off.

3) Heuristic based approaches such as [43], [14] develop energy-aware sensor nodes clustering using the signal strength values. However, it may affect the overall network LT. Thus, we use sensor nodes positions in the clustering process.

4) Dissimilar to the sensor nodes clustering approaches in [25], [29], ARSH-FATI-CHS applies to large-sized networks. Furthermore, ARSH-FATI-CHS does not produce any power overhead like the clustering approach presented in [26].

In order to compare the the performance of ARSH-FATICHS, we select three well-known clustering algorithms such as LEACH [24]. The reason behind selecting LEACH is that it is one of the classical clustering approach while PSO-C [18] and PSO-ECHS [14] are selected because these algorithms are the latest meta-heuristics for the same network scenario as we proposed in this paper.

Briefly we investigate an efficient and energy-aware sensor nodes clustering approach applicable to any-sized network based on ARSH-FATI-CHS meta-heuristic which is a population based algorithm. The ARSH-FATI-CHS is guided by A heuristic called NRC to enhance the overall LT of the network. Our developed fitness function considers residual energy, different distance parameters, and workload on the CHs during cluster formation.

\section{Preliminaries}

In this section we briefly discuss our energy and network model used for simulation purposes. We also define LT of a network which is used as a performance metric.

\section{A. Energy model}

We adopt the energy model used in [14], [44], [24], [18]. In this energy model the total energy consumption $(E)$ of a network is due to the energy dissipated by transmitter denoted by $E_{T X}(l, d)$ and receiver represented by $E_{R X}(l)$ given as follows.

$$
e_{\text {total }}(l, d)=E_{T X}(l, d)+E_{R X}(l)
$$

where $E_{T X}(l, d)$ is the energy consumed to run the power amplifier and radio electronics. Similarly, $E_{R X}(l)$ is the energy consumption of running the radio electronics. For each sensor node in the network, the energy consumed by the transmitter for transmitting $l$ bits is given as follows:

$$
E_{T X}(l, d)= \begin{cases}l \times E_{\text {elec }}+l \times e_{f s} \times d^{2} & d \leq d_{o} \\ l \times E_{\text {elec }}+l \times e_{m p} \times d^{4} & d \geq d_{o}\end{cases}
$$

where $E_{\text {elec }}$ is the energy consumed per bit for running the transmitter or receiver circuit, $e_{f s}$ and $e_{m p}$ represent the amplification energy for free space model and multi-path model while $d_{o}$ denotes the threshold transmission distance and generally its value is $d_{o}=\sqrt{\frac{e_{f s}}{e_{m p}}}$. The transmitter energy consumption is dependent on the distance parameter $d$ and the amount of data to be sent. If the data transmission distance is within the threshold range then transmittance energy is proportional to $d^{2}$ otherwise this relationship increases to $d^{4}$. Thus distance and workload play a significant role in the sensor nodes clustering for network life improvement.

Now the energy dissipation for the receiver to receive $l$ bit of data is estimated as follows:

$$
E_{R X}(l)=l \times E_{\text {elec }}
$$

where $E_{\text {elec }}$ is dependent on factors such as modulation, filtering, digital coding, and signal spreading.

\section{B. Network Model}

We consider a WSN that has $n$ number of sensor nodes and a BS. We adopt the wireless network model presented in [40], [14], [45], [12] and this scenario of the WSN possesses the following properties.

1) The sensor nodes are randomly distributed over a $2 D$ sensing field (that is xy plane) such that exactly one node exist at a point.

2) Sensor nodes are energy constrained and after deployment in the target area they are left unattended, thus battery recharge is impractical.

3) All the sensor nodes are homogeneous and possess similar processing and communication capabilities while consume same amount of energy for processing and communicating a data of $l$ bit.

4) After deploying the sensor nodes they are considered to be stationary with respect to the BS while each sensor node in the sensing field has the capability and equal probability to operate as a normal node or $\mathrm{CH}$.

5) Each sensor node has always a data ready to be sent to its $\mathrm{CH}$. The number of sensor nodes in the sensing field is always higher than the number of CHs.

6) The BS is considered to be situated inside of the WSN, while its position can be changed withing the sensing field.

7) In the clustered based WSN architecture, we assume that each $\mathrm{CH}$ aggregates the collected data and transmits this aggregated data to the BS.

8) The communication links among the sensor nodes and $\mathrm{CHs}$ are wireless and established when they are within the communication range of each other.

9) The sensor nodes can use various transmission power levels depending upon the distance to which the collected data to be sent.

\section{Node and Network Lifetime}

Before we describe the LT of the sensor network we define the LT of a sensor node and a $\mathrm{CH}$. The LT of a sensor node $s n_{i} \in S N$ when it chooses $c h_{j}$ as its $\mathrm{CH}$ is defined as follows: 


$$
L T\left(\operatorname{sn}_{i}, c h_{j}\right)=\left\lfloor\frac{e_{\text {residual }}^{i}}{e_{\text {total }}\left(l, d_{i}\right)}\right\rfloor
$$

where $d_{i}$ is the Euclidean distance between the $s n_{i}$ and $c h_{j}$ and $e_{\text {residual }}^{i}$ is the residual energy of $s n_{i}$.

The LT of a $\mathrm{CH}, c h_{j}$ is defined as follows:

$$
L T\left(c h_{j}\right)=\left\lfloor\frac{e_{\text {residual }}^{j}}{E_{T X}\left(l, d_{j}\right)+\sum_{s n_{i} \in \psi} E_{R X}(l)}\right\rfloor
$$

where $d_{j}$ is the euclidean distance between the $c h_{j}$ and Base Station (BS) and $\psi$ is the set of sensor nodes whose $\mathrm{CH}$ is $c h_{j}$.

There are are different definitions to describe the LT of a wireless network. However, the definition of LT used in this paper is given as follows:

LT Definition: LT of the network is the number of rounds until the First Node Dies (FND).

We use FND as a metric to describe the LT of a network and produce various simulations results based on this definition.

\section{ARSH-FATI BASEd Cluster HeAd SElECtion}

In this section we explain ARSH-FATI [23] algorithm and our CHs selection approach, ARSH-FATI-CHS for improving LT of the network and sensor nodes transmission energy consumption reduction.

\section{A. Terminologies}

In order to easily understand the proposed algorithm we add a list of the some important terminologies given as follows.

1) SNs: $\mathrm{A}$ set of sensor nodes i.e, $S N s=$ $\left\{s n_{1}, s n_{2}, \ldots, s n_{n}\right\}$.

2) CHs: A set of cluster heads, $C H s=\left\{c h_{1}, c h_{2} \ldots c h_{m}\right\}$ while $n>m$.

3) DR: Dimensional rate, taking on any value between the range $0<D R_{0} \leq 1$.

4) $\mu$ : The number of candidate solutions for the current population.

5) gBest: Member of the population with highest fitness value.

6) $\pi_{w}$ : Member of the population with lowest fitness value in all generation.

7) $\Delta f$ : The difference of the new and old fitness value.

8) $\lambda$ : Dimensional rate adaption parameter ranging, $0<$ $\lambda<1$.

9) $r$ : Random number ranging between 0 and 1.

10) $e$ : Representing energy.

11) $c h_{j}: j^{t h}$ cluster head in $m$ number of cluster heads.

12) $s n_{i}: i^{t h}$ sensor node in $n$ number of sensor nodes.

13) LT: Life time of the network

14) $\max G e n s:$ Maximum generation count.

\section{B. ARSH-FATI Algorithm Overview}

ARSH-FATI [23] is a population based algorithm in which, only the best and worst solutions of the previous population are used to generate the $\mu$ number of candidate solutions for the current population. Such kind of selection algorithms in literature are commonly referred to as $(1+\mu)$ selection algorithms. The robustness of ARSH-FATI algorithm lies in the notion of updating the parameter dimensional rate $(D R)$ at run-time during the searching process. ARSH-FATI algorithm attains a satisfactory trade-off between the exploitation and exploration attributes of the search process. The parameter $D R$ is the percentage of design variables that change to generate a new value for the member of the population. The strength of ARSH-FATI algorithm lies in $D R$ and the role of it is to adjust at run-time the exploitation and exploration features.

In this paper our objective is to maximize the minimum LT or in simpler words maximize the LT of the network. Therefore, the fitness function is the LT of the network:

$$
f=\min \left\{L T\left(s n_{i}, c h_{j}\right): \forall s n_{i} \in S N s\right\}
$$

where the $c h_{j}$ is the $\mathrm{CH}$ of sensor node $s n_{i}$.

Before we proceed with our discussion we define the following two terms:

1) Global Best, $g B e s t$, is a member of the population that has the highest fitness value $g$ BestFit in all generations.

2) Generation worst $\pi_{w}$ is a member of the population that has the lowest fitness value in a generation.

\section{ARSH-FATI-CHS}

The objective of ARSH-FATI-CHS described in Algorithm 1 is to find a set of $\mathrm{CHs}$ among the sensor nodes such that energy consumption reduces and LT of the network maximize. ARSH-FATI-CHS improves the network LT by maximizing the minimum LT in the network. The steps followed by ARSHFATI-CHS are explained as follows:

1) Setting the initial value of $\mathbf{D R}$ : We set $D R$ to and initial value $D R_{0}$ (Line 3$) . D R_{0}$ can take on any value between the range $0<D R_{0} \leq 1$. The higher $D R$ value means more explorative search that leads to large and unconstrained step sizes. Compared to this a small value of DR motivates a more exploitative search by allowing small and conservative steps in the search space. Therefore we set $D R_{0}=0.4$.

2) Population Generation: We initially generate a matrix $\Pi$ of dimensions $\mu \times m$ of zeros (Line 1 ), where $\mu$ is the size of the population and $m$ is the total number of CHs. We use the notation $\Pi[i,:]$ to access the $i^{t h}$ member of the population. Each member of the population has $m$ elements and the notation $\Pi[i, j]$ is used to access the $j^{\text {th }}$ element of $i^{\text {th }}$ member. The value of an element is a positive integer that indicates a sensor node chosen as a $\mathrm{CH}$. For example if the value of an element is 2 this reflects that sensor node $s n_{2}$ is selected as a $\mathrm{CH}$. Each member of the population reflects one possible $\mathrm{CH}$ selection and the entire population reflects $\mu$ different $\mathrm{CH}$ selections.

We generate and initial value of the member of the population by randomly selecting $m \mathrm{CHs}$ among the sensor nodes and its fitness value is calculated by executing the clustering algorithm 2 . We repeat this for the other members of the population to generate an initial population (Lines 4-7). 
3) Population Refinement: We refine the initial population until the termination criteria satisfy (Lines 10-28). In each generation we update each member of the population. The $j^{t h}$ element of the $i^{t h}$ member is updated as follows:

$$
\Pi[i, j] \leftarrow \begin{cases}\min \left(a b s \left(\left\lceil\Pi[i, j]+r_{1}(g B e s t[i]-\right.\right.\right. & \\ \left.\left.\left.\Pi[i, j])-r_{2}\left(\pi_{w}[i]-\pi[i]\right)\right)\right), m\right) & \text { if } r \leq D R \\ \Pi[i, j] & \text { otherwise }\end{cases}
$$

where $r_{1}$ and $r_{2}$ are the random numbers. The term $r_{1}(g B e s t[i]-\Pi[\eta, i])$ reflects the likelihood that the member moves closer to global best and the term $r_{2}\left(\pi_{w}[i]-\Pi[\eta, i]\right)$ reflects the likelihood that it moves away from the worst member of the population.

We use an acceptance probability function $P(\Delta f, T)$ to adopt or reject the new value of the member $\Pi[i,:]$ (Lines 18-24). The parameter $\Delta f$ is the difference of the new and old fitness value of $\Pi[i,:], \Delta f=f_{\text {new }}-f_{\text {old }}$. The parameter $T$ is referred to as temperature. We define function $P(\Delta f, T)$ as follows:

$$
P(\Delta f, T)= \begin{cases}1 & \text { if } f_{\text {new }}>f_{\text {old }} \\ e^{\frac{\Delta f}{T}} & \text { otherwise }\end{cases}
$$

when the new $\mathrm{CH}$ selection increase the network LT it is always accepted. If the new $\mathrm{CH}$ selection is worse than the current selection, probability still exists that may be accepted. We have included this feature in ARSH-FATICHS to prevent it getting stuck in a local optimum. The value of temperature $T$ reduces in each iteration by multiplying it with a cooling factor $\lambda_{1},\left(0<\lambda_{1}<1\right)$ (Line 27). The value of $\lambda_{1}$ is calculated from maxGens:

$$
\lambda_{1}=\left(\frac{T_{1}}{T_{2}}\right)^{\frac{1}{\max G e n s}}
$$

where $T_{2}$ is a very large number and $T_{1}$ is a very small number. Initially the value of temperature is set to $T_{2}$ and it reduces to $T_{1}$ as optimization finishes.

In each generation we update $D R$ (Line 25):

$$
D R= \begin{cases}\min \left\{\frac{D R}{\lambda}, D R_{\max }\right\} & \text { if } g \text { Best improves } \\ \max \left\{\lambda D R, D R_{\min }\right\} & \text { otherwise }\end{cases}
$$

where the $\lambda$ is dimensional rate adaption parameter and its value lies within the range $0<\lambda<1$. In this work $\lambda$ is set to 0.98 . The parameter $\lambda$ sets the new value of the $D R$ during the optimization process.

The values of $D R_{\min }$ and $D R_{\max }$ are respectively the upper and lower bounds on $D R$. The values of $D R_{\min }$ and $D R_{\max }$ must be set subject to constraint $0<D R_{\min }<D R_{\max }<1$. We avoid excessive exploration and exploitation by setting $D R_{\min }$ and $D R_{\max }$ to 0.2 and 0.6 respectively.

4) Termination criteria: ARSH-FATI-CHS terminates if either the generation count reaches maximum generation maxGens or no improvement is observed in gBest for consecutive $\beta$ generations.

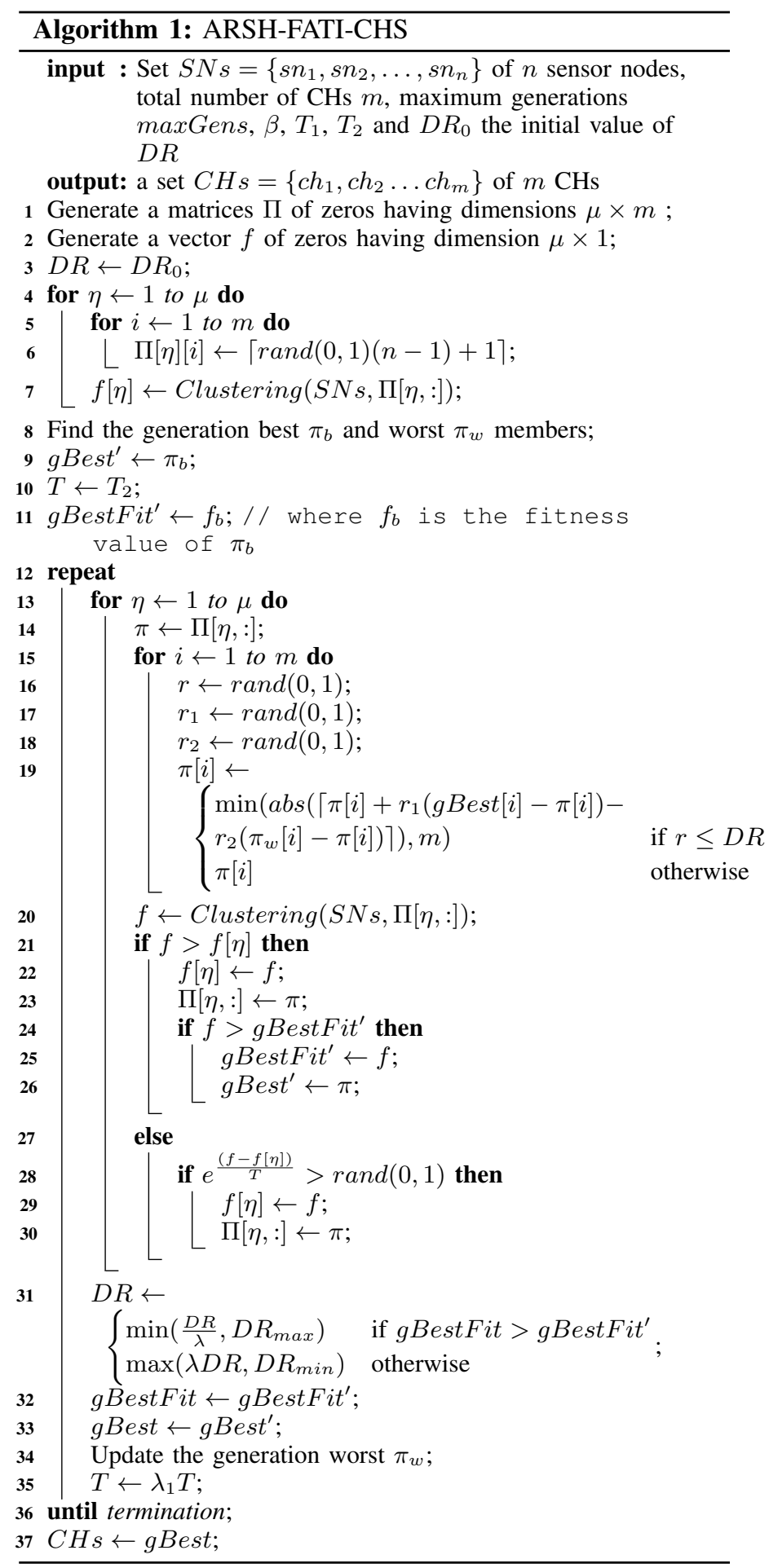

\section{Cluster Formation}

In this section we describe our cluster formation technique. Given a set of $\mathrm{CHs}$ the objective of our cluster formation approach is to maximize the network LT. Algorithm 2 describes our cluster formation approach. It is based on the ranking function $\operatorname{Rank}\left(s n_{i}, c h_{j}\right)$ that sensor nodes use to choose a $\mathrm{CH}$. The ranking function is based on the following parameters:

1) $s n_{i}$ residual energy: the larger the residual energy of 
the sensor node larger its LT. Therefore,

$$
\operatorname{Rank}\left(s n_{i}, c h_{j}\right) \propto e_{\text {residual }}^{i}
$$

2) $s n_{i}$ total energy: the sensor node energy consumption negatively impacts its LT. The sensor node should join the $\mathrm{CH}$ such that its transmission energy minimizes. Therefore,

$$
\operatorname{Rank}\left(s n_{i}, c h_{j}\right) \propto \frac{1}{e_{\text {total }}\left(l, d_{i}\right)}
$$

3) $c h_{j}$ residual energy: the sensor node $s n_{i}$ should join the $\mathrm{CH}$ that has large residual energy. Therefore,

$$
\operatorname{Rank}\left(s n_{i}, c h_{j}\right) \propto e_{\text {residual }}^{j}
$$

4) $c h_{j}$ total energy: the $\mathrm{CH}$ total energy consumption negatively impacts its LT. Therefore,

$$
\operatorname{Rank}\left(s n_{i}, c h_{j}\right) \propto \frac{1}{e_{\text {total }}\left(l, d_{j}\right)}
$$

Combining equations (10), (11), (12) and (13) we get the following function:

$$
\begin{gathered}
\operatorname{Rank}\left(s n_{i}, c h_{j}\right) \propto \frac{e_{\text {residual }}^{i} e_{\text {residual }}^{j}}{e_{\text {total }}\left(l, d_{i}\right) e_{\text {total }}\left(l, d_{j}\right)} \\
\operatorname{Rank}\left(s n_{i}, c h_{j}\right)=\kappa\left\lfloor\frac{e_{\text {residual }}^{i}}{e_{\text {total }}\left(l, d_{i}\right)}\right\rfloor\left\lfloor\frac{e_{\text {residual }}^{j}}{e_{\text {total }}\left(l, d_{j}\right)}\right\rfloor \\
\operatorname{Rank}\left(s n_{i}, c h_{j}\right)=\kappa L T\left(s n_{i}, c h_{j}\right) L T\left(c h_{j}\right)
\end{gathered}
$$

where $\kappa$ is the constant of proportionality. We assume $\kappa=1$ in this paper without any loss of generality.

Algorithm 2 describes our cluster formation approach. It is a greedy heuristic algorithm that at each stage finds a set of valid choices and makes a locally optimal choice.

The following two steps repeat until each sensor node has chosen a $\mathrm{CH}$ :

1) Step 1 (Search): Each sensor node that has not joined a $\mathrm{CH}$ tentatively selects one by one each $\mathrm{CH}$ and calculates the rank using equation (16) (Lines 3-6). Then find the sensor node $s n_{i}$ and $\mathrm{CH} c h_{j}$ pair that has the maximum rank value.

2) Step 2 (Choose): The sensor node $s n_{i}$ joins $\mathrm{CH} c h_{j}$

\section{EXPERIMENTAl SetuP AND Results}

In this section, we explain our experimental setup used for simulation. We also generate different results considering various scenarios. The results are compared with state-of-theart clustering approaches to prove the superiority of ARSHFATI-CHS over other existing technique.

\section{A. Experimental Setup}

We built the simulation environment in Python version 3.7.3 moreover, we conducted the experiments using hardware platform of Intel (R) Xeon (R), i5-3570 CPU with the clock frequency of $3.50 \mathrm{GHz}$ and $16.00 \mathrm{~GB}$ memory, $10 \mathrm{MB}$ cache. We perform the simulation for different number of sensor nodes various percentage of $\mathrm{CHs}$ at different locations of BS in the sensing field of area $200 \times 200 \mathrm{~m}^{2}$. The sensor nodes

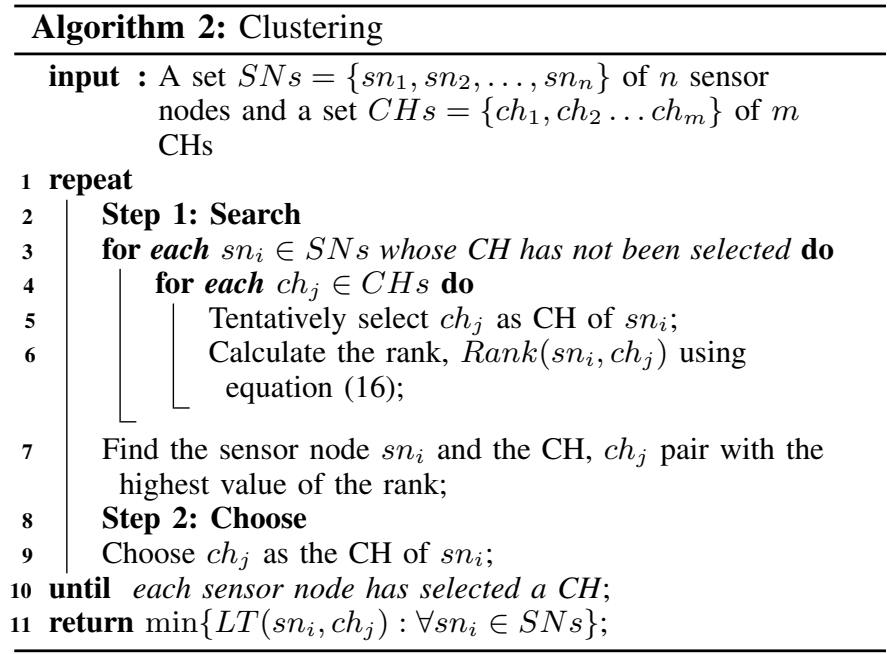

change within a range of 100 to 400 and the number of selected CHs vary from $15 \%$ to $30 \%$ while BS is positioned at (50, $50),(100,100),(150,150)$. We assume that each sensor node carries equal initial residual energy of $2 \mathrm{~J}$. Other constant parameters used in this paper for simulations are enlisted in TABLE (I) adopted from [24], [14], [18].

TABLE (I) Various parameters used in simulation

\begin{tabular}{ll}
\hline \multicolumn{1}{c}{ Parameter } & \multicolumn{1}{c}{ Value } \\
\hline Area of the Network & $200 \times 200 \mathrm{~m}^{2}$ \\
Base Station Location & $(50,50),(100,100),(150,150)$ \\
Number of Sensor Nodes & 100 to 400 \\
Initial Sensor Node Energy & $2.0 \mathrm{~J}$ \\
$E_{\text {elec }}$ & $50 \mathrm{~nJ} / \mathrm{bit}$ \\
$e_{f s}$ & $10 \mathrm{pJ} / \mathrm{bit} / \mathrm{m}^{2}$ \\
$e_{m p}$ & $0.0013 \mathrm{pJ} / \mathrm{bit} / \mathrm{m}^{4}$ \\
$d_{\max }$ & $100 \mathrm{~m}$ \\
$d_{o}$ & $30 \mathrm{~m}$ \\
Packet size & $4000 \mathrm{bits}$ \\
Percentage of CHs & $10 \%$ to $30 \%$ \\
\hline
\end{tabular}

We compare the results of our proposed $\mathrm{CHs}$ selection approach, ARSH-FATI-CHS with a PSO based state-of-the-art technique as a baseline developed by Rao et al. [14]. In their energy-aware clustering approach they consider parameters such as intra-cluster distance, residual energy of the sensor node, and sink distance in the fitness function. The network LT is increased by developing a PSO based energy-efficient $\mathrm{CH}$ selection (PSO-ECHS). The PSO-ECHS determines the optimal positions of a pre-determined numbers of $\mathrm{CHs}$ for a set of sensor nodes distributed over a specific area of the sensing fields. The non-CH sensor nodes in the field join their elected $\mathrm{CHs}$ based on a derived weight function. We also compare the performance of ARSH-FATI-CHS with the widely used LEACH [24] and PSO-C [18]. As a performance metric we consider LT to compare ARSH-FATI-CHS with other existing approaches. LT is the measure of the number of rounds when the first sensor nodes dissipates all of its energy.

\section{B. Results and Discussions}

We consider different scenarios for our simulations to generate various results. The list of abbreviations used in results are 
TABLE (II) List of abbreviations used in results

\begin{tabular}{ll}
\hline Parameter & \multicolumn{1}{c}{ Description } \\
\hline TA & Target Area \\
BS & Base Station \\
NSNs & Number of Sensor Nodes \\
NCHs & Number of Cluster Heads \\
FND & First Node Death \\
ISNE & Initial Sensor Node Energy \\
NoR & Number of Rounds \\
NoANs & Number of Alive Nodes \\
IoBSL & Impact of Base Station Location \\
NoPR & Number of Packets Received \\
IoNCHs & Impact of Number of Cluster Heads \\
\hline
\end{tabular}

listed in TABLE (II). The parameter such as NSNs is changed in the sensing field/target area and results are produced. We also empirically observe IoBSL and IoNCHs in the results.

1) Scenario 1(NoR): In this scenario we set $T A=200 \times$ $200 \mathrm{~m}^{2}, N S N s=(100,200,300,400), I S N E=2.0 \mathrm{~J}$, $B S=(50,50)$, and $N C H s=(10 \%, 15 \%, 20 \%, 25 \%)$ while we compare the performance of ARSH-FATI-CHS combined with NRC in terms of $N o R$ after $F N D$ in the network. This scenario basically describes the LT of the network. LT is an important metric to evaluate the performance and efficiency of an algorithm as discussed in detail by wang et al. [46].

Fig. (2) demonstrates the performance improvement of ARSH-FATI-CHS integrated with NRC over the existing approaches. A set of different sensor nodes are used for simulations such as, Fig. (2)(a) deploys $N S N s=100$, similarly, Fig. (2)(b) uses $N S N s=200$ while Fig (2)(c) and Fig. (2)(d) deploy $N S N s=300$ and $N S N s=400$ respectively. ARSH-FATI-CHS outperforms clustering approaches such as LEACH, PSO-C, and PSO-ECHS. ARSH-FATI-CHS achieves an average improvement of $\sim 60 \%, \sim 40 \%$, and $\sim 25 \%$ over LEACH, PSO-C, and PSO-ECHS respectively in terms of NoR after FND. This improvement of ARSH-FATI-CHS over LEACH occurs because LEACH is a probabilistic approach and randomly selects $\mathrm{CHs}$ which results into uneven distribution of clusters so, increasing the energy consumption of the network while reducing the overall performance i.e. LT of the network. Moreover, LEACH can select a sensor node with least residual energy as a $\mathrm{CH}$ thus, it adversely impacts the NoR after FND. Contrarily our fitness function in ARSH-FATI-CHS considers the residual energy, different distance parameters, and work load on the $\mathrm{CHs}$ during the cluster formation. Similarly, ARSH-FATI-CHS outperforms population based clustering approach, PSO-C. Though, PSO-C improves the intra-cluster distance i.e. the Euclidian distance of the sensor nodes to their selected $\mathrm{CHs}$ during cluster formation but neglects the workload on them. Compared to PSO-ECHS our proposed clustering approach ARSH-FATICHS achieves higher NoR after FND. ARSH-FATI-CHS also performs better solution space exploration during the cluster formation. Moreover, it is stagnation controlled meta-heuristic and determines the global optimal solution. In other words ARSH-FATI-CHS efficiently maximizes the minimum LT of a sensor node in the network. ARSH-FATI-CHS associates a sensor node having the least residual energy to a $\mathrm{CH}$ such that it consumes minimal possible transmission energy for data transmission. Concisely, ARSH-FATI-CHS performs better than LEACH, PSO-C, and PSO-ECHS in terms of NoR after FND when different sensor nodes and $\mathrm{CHs}$ are deployed in the network.

2) Scenario 2(NoANs): In this scenario 2 we set the parameters as $T A=200 \times 200 \mathrm{~m}^{2}, N S N s=(100,200,300,400)$, $I S N E=2.0 \mathrm{~J}, B S=(50,50)$, and $N C H s=25 \%$. We observe the performance of the ARSH-FATI-CHS in terms of NoANs. Specifically this scenario describes the amount of alive nodes after certain number of rounds.

Fig. (3) shows the performance analysis of ARSH-FATICHS integrated with NRC over LEACH, PSO-C, and PSOECHS in terms of NoANs. The x-axis represents NoR and y-axis denotes the NoANs. Fig. (3)(a) and Fig. (3)(b) show the NoANs comparison for $N S N s=100$ and $N S N s=200$. Similarly Fig. (3)(c) and Fig. (3)(d) represent the simulation results for $N S N s=300$ and $N S N s=400$ respectively. ARSH-FATI-CHS significantly improves the NoANs when compared to LEACH, PSO-C, and PSO-ECHS. ARSH-FATICHS attains an average efficiency of $\sim 60 \%, \sim 40 \%$, and $\sim 25 \%$ over LEACH, PSO-C, and PSO-ECHS respectively. Unlike LEACH, ARSH-FATI-CHS performs reclustering when death of a $\mathrm{CH}$ occurs and associates the sensor nodes to other $\mathrm{CHs}$ in the network. On the contrary the biggest drawback of LEACH is that when a $\mathrm{CH}$ dies then that particular cluster becomes useless and the gathered data would never reach to the destination i.e. BS. Furthermore, LEACH may select a $\mathrm{CH}$ at the boundary of the network which potentially can lead to the improper clustering resulting performance degradation. Moreover, ARSH-FATI-CHS also performs uniform distribution of $\mathrm{CHs}$. Furthermore, as the NoR increases the residual energy of the sensor decreases. Subsequently, effective $\mathrm{CHs}$ selection plays an important role to increase the LT of the network. Our meta-heuristic performs efficient $\mathrm{CHs}$ selection using a novel fitness function. Therefore, our algorithm ARSH-FATI-CHS significantly improves the NoANs compared to LEACH. Now, compared to PSO$\mathrm{C}$ our meta-heuristic produces better results because of our fitness function not only considers the intra-cluster distance but also the workload on the CHs. Similarly, it outperforms PSO-ECHS by dynamically switching between an exploitative and exploitative search modes to achieve better solution space search. Concisely, though ARSH-FATI-CHS is a simple but yet effective approach for $\mathrm{CHs}$ selection and enhancing the overall LT of the network.

3) Scenario 3(NoPR): In this scenario we set $T A=$ $200 \times 200 \mathrm{~m}^{2}, N S N s=(100,200,300,400), I S N E=2.0$ $\mathrm{J}, B S=(0,0),(50,50),(100,100),(150,150)$, and $N C H s$ $=10 \%$ while we compare ARSH-FATI-CHS performance in terms of $N o P R$ at the BS.

Fig. (4) illustrates the performance comparison of ARSHFATI-CA with other state-of-the-art approaches in terms of NoPR at the BS. We set different $N S N s$ at various positions of the BS. Fig. (4)(a) uses $N S N s=100$, similarly, Fig. (4)(b) deploys $N S N s=200$ while Fig (4)(c) and Fig. (4)(d) use $N S N s=300$ and $N S N s=400$ respectively. The horizontal axis shows position of the BS and vertical axis represents NoPR. ARSH-FATI-CA outperforms LEACH, 


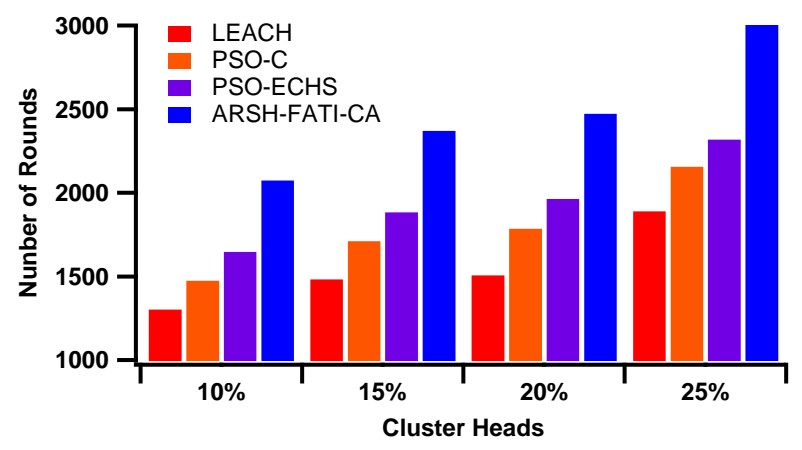

(a) NoR after FND at NSNs $=100$

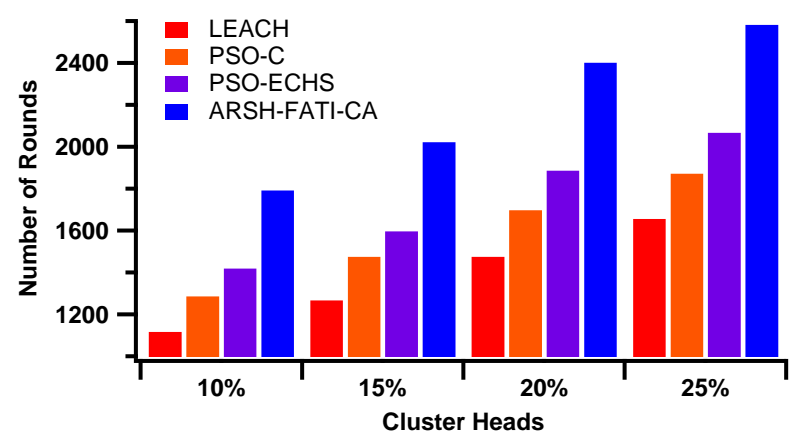

(c) NoR after FND at NSNs $=300$

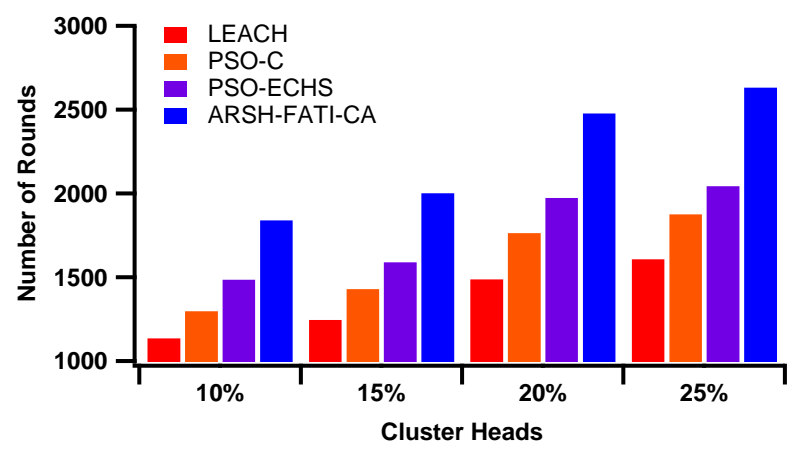

(b) NoR after FND at NSNs $=200$

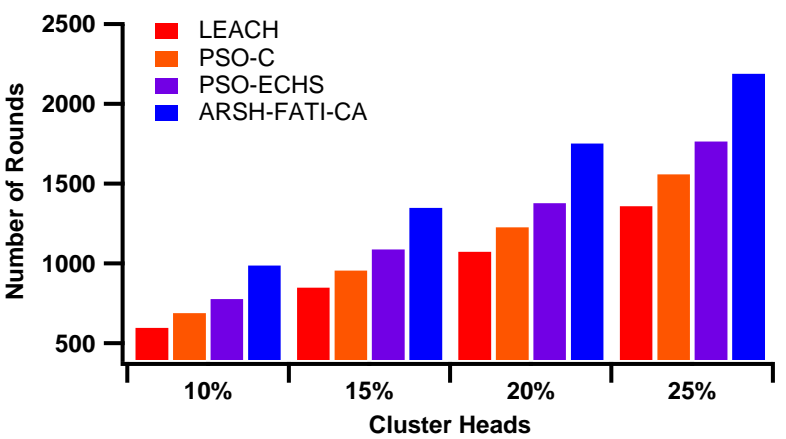

(d) NoR after FND at NSNs $=400$

Fig. (2) Performance analysis in terms of NoR after FND using different NSNs

PSO-C, and PSO-ECHS and achieves higher NoPR at the BS. This improvement of ARSH-FATI-CA in terms of NoPR over other existing clustering approaches is due performing better energy-efficient clustering. Consequently, it enhances the LT of the network and lower energy is consumed which increases the NoPR at the BS. It is also worth noticing that NoPR at the BS also depends on the position of the BS in the network. Higher NoPR is achieved when BS is positioned at $(100,100)$ i.e. centre of the target area. NoPR decreases significantly when $\mathrm{BS}$ is placed away from the centre. The $\mathrm{CHs}$ consume more energy to transmit the aggregated data to the BS when it is positioned at the corners of the target area thus NoPR reduces. Concisely, the optimal position of the BS is centre of the target area where highest NoPR can be achieved. The impact of the BS position is further investigated in Scenario 4(IoBSL and IoNCHs).

4) Scenario 4(IoBSL and IoNCHs): We set the parameters as $T A=200 \times 200 \mathrm{~m}^{2}, N S N s=(100,200,300,400)$, and $I S N E=2.0 \mathrm{~J}$. In this scenario 4 we observe the impact of the BS location and variations in $\mathrm{CHs}$ percentage on NoR after FND. These two specific cases are discussed as follows:

1) First we consider $N C H s=25 \%$ and $B S=(50,50)$, (100, 100), (150, 150) for observing IoBSL on NoR after FND. Fig. (5)(a) demonstrates IoBSL on NoR after FND. The horizontal axis shows NSNs and the vertical axis represents NoR. The NoR depends on the position of the BS. The NoR significantly increases when BS is positioned at the center of the network i.e. $(100,100)$. The NoR decreases when BS is moved towards positions $(50,50)$ and $(150,150)$. This performance degradation occurs because data packets from the selected $\mathrm{CHs}$ travel longer distance when $\mathrm{BS}$ is positioned at the coroner of the network. Thus, transmitting the gathered data for a longer distance adversely affects the LT and therefore, FND occurs at lower NoR. An average $~ 70 \%$ improvement in terms of NoR after FND occurs when $\mathrm{BS}$ is placed at the centre compared to when BS is positioned at the corners of the network. The bottom line is that the ideal place of BS is the centre of the network.

2) Second to analyze the IoNCHs on network performance we consider $B S=(50,50)$ and $N C H s=10 \%, 15 \%$, $20 \%, 25 \%, 30 \%$ as shown in Fig. (5)(b). The $\mathrm{x}$-axis denotes NSNs while y-axis represents NoR after FND. A significant improvement in NoR occurs when percentage of $\mathrm{CHs}$ is increased. The NoR increases until reaching $\mathrm{CHs}=25 \%$ while starts decreasing at $\mathrm{CHs}=30 \%$. It is a proven fact that $\mathrm{CHs}$ usually consume more energy as compared to non-CHs because they collect data from the sensor nodes within the cluster and transmit it to the BS [14]. If lower percentage of $\mathrm{CH}$ are used then clusters with higher NSNs are formed. This increases the receiving and transmission energy of the $\mathrm{CH}$ and there is higher chance of dying quickly. Therefore, NoR increases when higher number of $\mathrm{CHs}$ are used in the network. In result it reduces the workload on the $\mathrm{CHs}$ and prolongs the overall LT of the network. An average $\sim 25 \%$ improvement in NoR is observed when $C H s=15$ is changed to $C H s=25$ in the network. It is also worth noticing that after certain percentage of $\mathrm{CHs}$ 


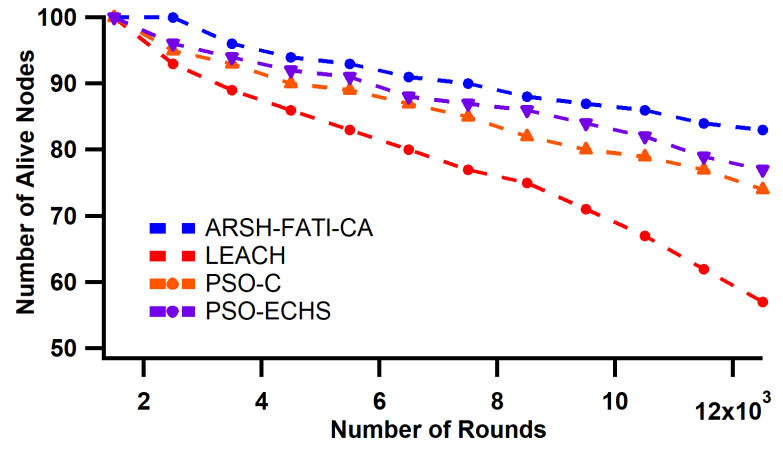

(a) NoAN at NSNs $=100$

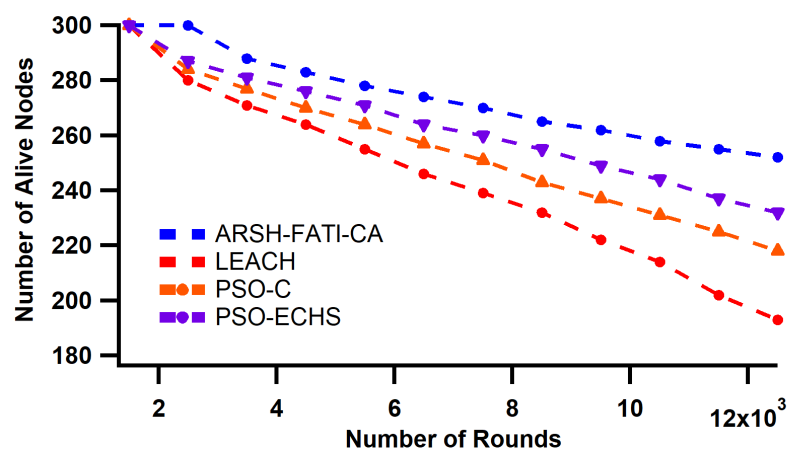

(c) NoAN at NSNs $=300$

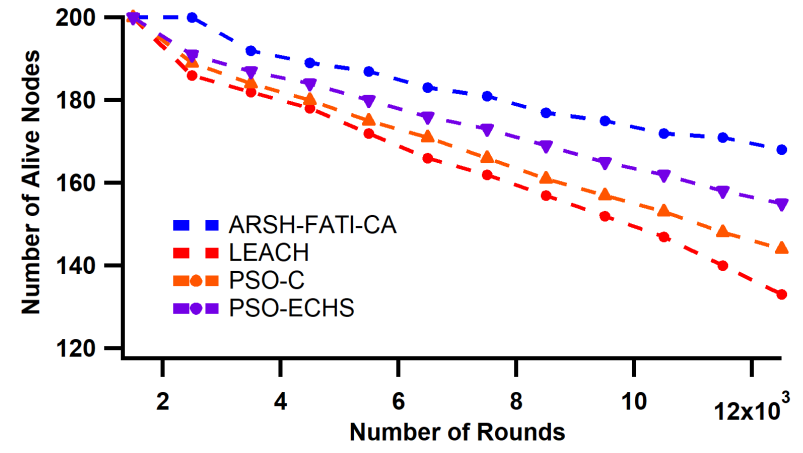

(b) NoAN at NSNs $=200$

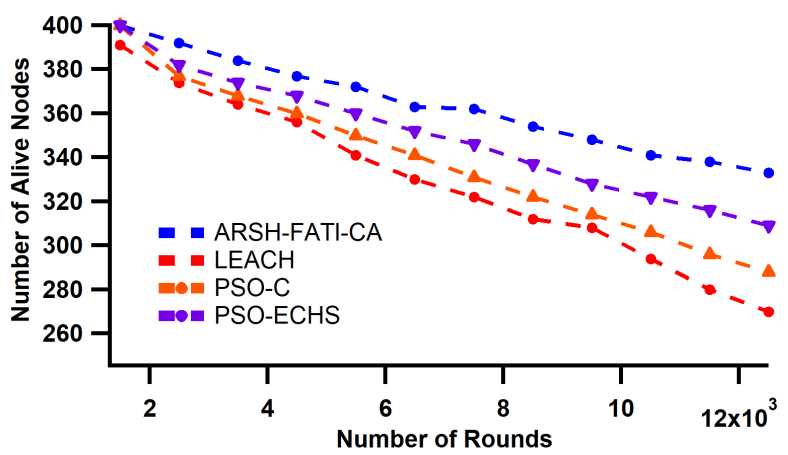

(d) NoAN at NSNs $=400$

Fig. (3) Performance analysis in terms of NoAN using different NSNs and $\mathrm{NCHs}=25 \%$ at $\mathrm{BS}=(50,50)$

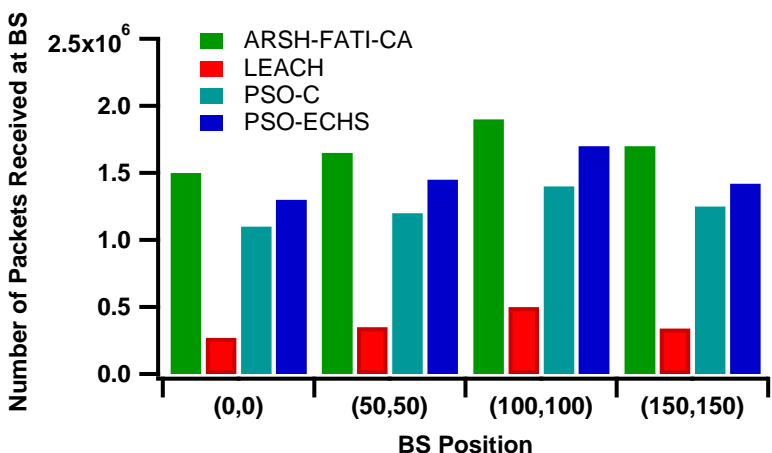

(a) NoPR at NSNs $=100$

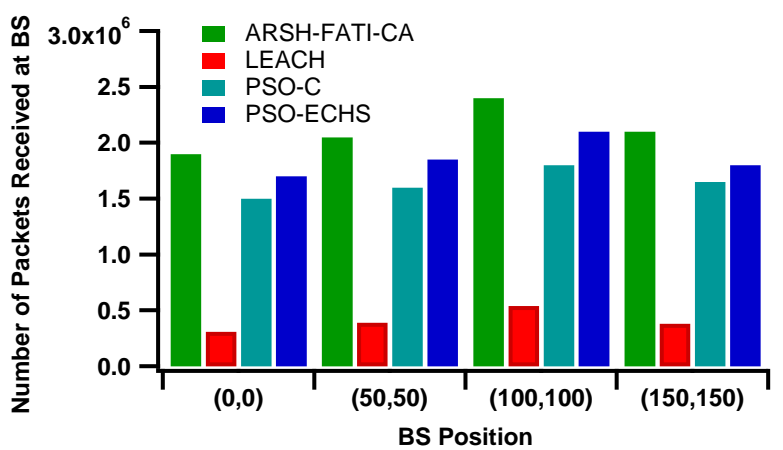

(c) NoPR at NSNs $=300$

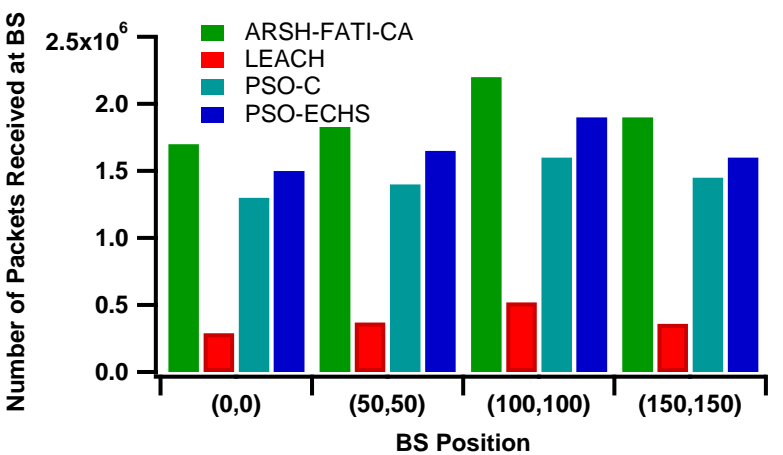

(b) NoPR at NSNs $=200$

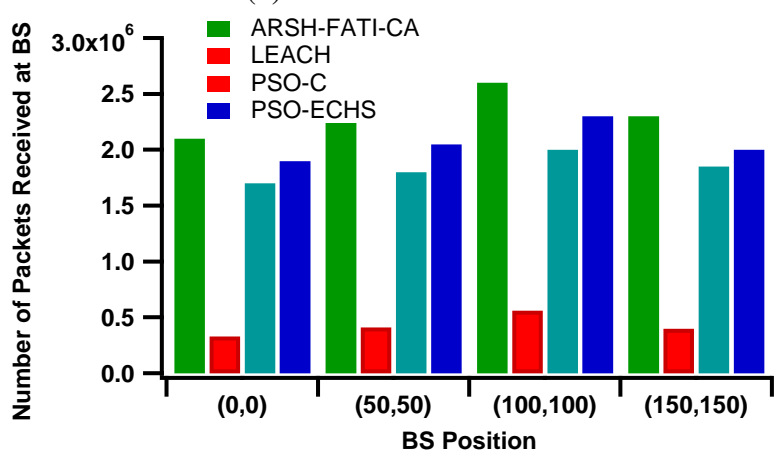

(d) NoPR at NSNs $=400$

Fig. (4) Performance comparison in terms of NoPR using $\mathrm{NCHs}=10 \%$ at different NSNs and BS positions 


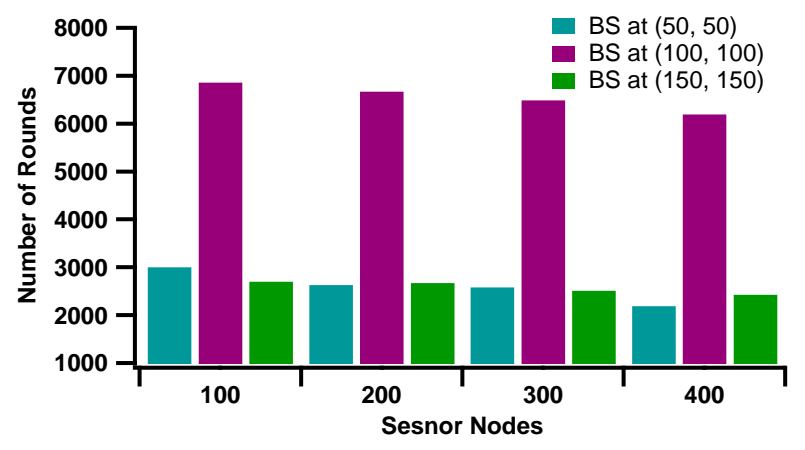

(a) Impact of base station

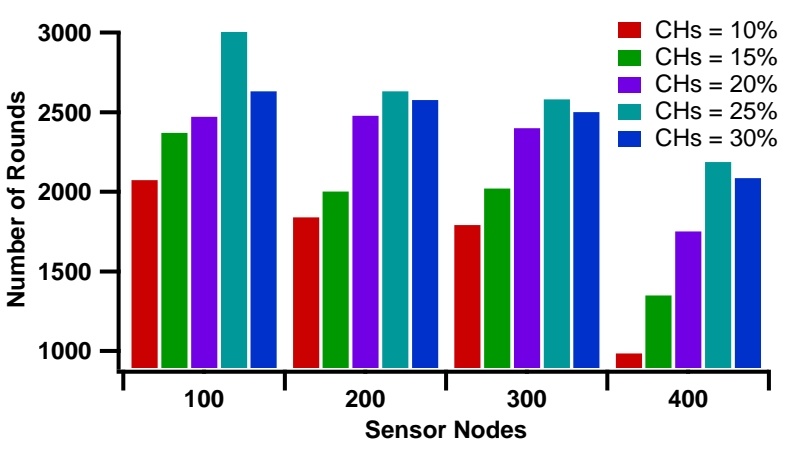

(b) Impact of cluster heads

Fig. (5) Impact of base station and cluster heads on LT of the network

despite of improvement in NoR reduction occurs. This decrease in NoR is due to the fact that the number of transmissions to the BS increases which can adversely affect the NoR. Subsequently, an optimal number of CHs are required to maximize the overall NoR and LT.

TABLE (III) ARSH-FATI-CHS performance improvement comparison

\begin{tabular}{lc}
\hline Clustering Technique & ARSH-FATI-CHS \\
\hline LEACH & $60 \%$ \\
PSO-C & $40 \%$ \\
PSO-ECHS & $25 \%$ \\
\hline
\end{tabular}

Concisely we achieve an overall average LT improvement of $\sim 25 \%$, 40\%, $60 \%$ over LEACH, PSO-C, and PSOECHS when ARSH-FATI-CHS integrated with NRC heuristic is used for cluster formation. Moreover, we also observed that the LT of the network also depends on the position of the BS and the percentage of CHs deployed in the network. The LT performance of ARSH-FATI-CHS over other existing techniques is summarized in TABLE (III).

\section{CONCLUSION}

Transmission energy consumption reduction is one of the major concerns in designing clustering algorithms for largesized Wireless Sensor Networks (WSNs). The existing population based meta-heuristics are complex and need different parameters tuning to achieve higher energy-efficiency. Furthermore, state-of-the-art clustering approaches neglect to consider residual energy of the nodes, different distance parameters, and workload on the Cluster Heads (CHs) to enhance LT of the network. In this paper we developed ARSH-FATI based Cluster Head Selection (ARSH-FATI-CHS) integrated with a heuristic called Novel Ranked based Clustering (NRC) for efficient cluster formation to enhance the overall LT of the network. ARSH-FATI-CHS is a simple yet effective clustering algorithm that dynamically switches between the exploitative and explorative search modes for achieving higher performance trade-off. Our fitness function considers various parameters such as residual energy, node distance, base station distance location, and work load on the CHs. The experimental results show that ARSH-FATI-CHS outperforms the existing population based clustering algorithms in terms of network
LT enhancement. ARSH-FATI-CHS achieves an average LT improvement of $60 \%, 40 \%$, and $25 \%$ over LEACH, PSO$\mathrm{C}$, and PSO-ECHS. Furthermore, we also investigated that position of Base Station (BS) and percentage of CHs also play an important role to improve the overall LT of the network.

\section{REFERENCES}

[1] D. E. Boubiche, A.-S. K. Pathan, J. Lloret, H. Zhou, S. Hong, S. O. Amin, and M. A. Feki, "Advanced industrial wireless sensor networks and intelligent iot," IEEE Communications Magazine, vol. 56, no. 2, pp. 14-15, 2018.

[2] Z. Cui, Y. Cao, X. Cai, J. Cai, and J. Chen, "Optimal leach protocol with modified bat algorithm for big data sensing systems in internet of things," Journal of Parallel and Distributed Computing, vol. 132, pp. 217-229, 2019.

[3] Y.-W. Lin, Y.-B. Lin, M.-T. Yang, and J.-H. Lin, "Ardutalk: An arduino network application development platform based on iottalk," IEEE Systems Journal, vol. 13, no. 1, pp. 468-476, 2017.

[4] H. Ali, U. U. Tariq, Y. Zheng, X. Zhai, and L. Liu, "Contention $\&$ energy-aware real-time task mapping on noc based heterogeneous mpsocs," IEEE Access, vol. 6, pp. 75 110-75 123, 2018.

[5] L. Atzori, A. Iera, and G. Morabito, "The internet of things: A survey," Computer networks, vol. 54, no. 15, pp. 2787-2805, 2010.

[6] J. Gubbi, R. Buyya, S. Marusic, and M. Palaniswami, "Internet of things (iot): A vision, architectural elements, and future directions," Future generation computer systems, vol. 29, no. 7, pp. 1645-1660, 2013.

[7] T. Rault, A. Bouabdallah, and Y. Challal, "Energy efficiency in wireless sensor networks: A top-down survey," Computer Networks, vol. 67, pp. 104-122, 2014.

[8] S. S. Iyengar, H.-C. Wu, N. Balakrishnan, and S. Y. Chang, "Biologically inspired cooperative routing for wireless mobile sensor networks," IEEE Systems Journal, vol. 1, no. 1, pp. 29-37, 2007.

[9] O. Bello and S. Zeadally, "Intelligent device-to-device communication in the internet of things," IEEE Systems Journal, vol. 10, no. 3, pp. 1172-1182, 2014.

[10] A. Aliyu, A. H. Abdullah, O. Kaiwartya, Y. Cao, J. Lloret, N. Aslam, and U. M. Joda, "Towards video streaming in iot environments: Vehicular communication perspective," Computer Communications, vol. 118, pp. 93-119, 2018.

[11] V. C. Gungor and G. P. Hancke, "Industrial wireless sensor networks: Challenges, design principles, and technical approaches," IEEE Transactions on industrial electronics, vol. 56, no. 10, pp. 4258-4265, 2009.

[12] G. P. Gupta and S. Jha, "Integrated clustering and routing protocol for wireless sensor networks using cuckoo and harmony search based metaheuristic techniques," Engineering Applications of Artificial Intelligence, vol. 68, pp. 101-109, 2018.

[13] F. K. Shaikh, S. Zeadally, and E. Exposito, "Enabling technologies for green internet of things," IEEE Systems Journal, vol. 11, no. 2, pp. 983-994, 2015.

[14] P. S. Rao, P. K. Jana, and H. Banka, "A particle swarm optimization based energy efficient cluster head selection algorithm for wireless sensor networks," Wireless networks, vol. 23, no. 7, pp. 2005-2020, 2017. 
[15] G. P. Gupta, M. Misra, and K. Garg, "Energy and trust aware mobile agent migration protocol for data aggregation in wireless sensor networks," Journal of Network and Computer Applications, vol. 41, pp. 300-311, 2014.

[16] F. K. Shaikh and S. Zeadally, "Energy harvesting in wireless sensor networks: A comprehensive review," Renewable and Sustainable Energy Reviews, vol. 55, pp. 1041-1054, 2016.

[17] I. F. Akyildiz, W. Su, Y. Sankarasubramaniam, and E. Cayirci, "Wireless sensor networks: a survey," Computer networks, vol. 38, no. 4, pp. 393 422,2002

[18] N. A. Latiff, C. C. Tsimenidis, and B. S. Sharif, "Energy-aware clustering for wireless sensor networks using particle swarm optimization," in 2007 IEEE 18th International Symposium on Personal, Indoor and Mobile Radio Communications. IEEE, 2007, pp. 1-5.

[19] S. K. Gupta and P. K. Jana, "Energy efficient clustering and routing algorithms for wireless sensor networks: Ga based approach," Wireless Personal Communications, vol. 83, no. 3, pp. 2403-2423, 2015.

[20] A. Mohajerani and D. Gharavian, "An ant colony optimization based routing algorithm for extending network lifetime in wireless sensor networks," Wireless Networks, vol. 22, no. 8, pp. 2637-2647, 2016.

[21] P. Kuila and P. K. Jana, "A novel differential evolution based clustering algorithm for wireless sensor networks," Applied soft computing, vol. 25 , pp. $414-425,2014$.

[22] H. Zhang, S. Zhang, and W. Bu, "A clustering routing protocol for energy balance of wireless sensor network based on simulated annealing and genetic algorithm," International Journal of Hybrid Information Technology, vol. 7, no. 2, pp. 71-82, 2014.

[23] U. Ullah Tariq, H. Ali, L. Liu, J. Panneerselvam, and X. Zhai, "Energyefficient static task scheduling on vfi based noc-hmpsocs for intelligent edge devices in cyber-physical systems," ACM Transactions on Intelligent Systems and Technology, 2019.

[24] W. R. Heinzelman, A. Chandrakasan, and H. Balakrishnan, "Energyefficient communication protocol for wireless microsensor networks," in Proceedings of the 33rd annual Hawaii international conference on system sciences. IEEE, 2000, pp. 10-pp.

[25] S. Lindsey and C. S. Raghavendra, "Pegasis: Power-efficient gathering in sensor information systems," in Proceedings, IEEE aerospace conference, vol. 3. IEEE, 2002, pp. 3-3.

[26] V. Loscri, G. Morabito, and S. Marano, "A two-levels hierarchy for low-energy adaptive clustering hierarchy (tl-leach)," in IEEE vehicular technology conference, vol. 62, no. 3. IEEE; 1999, 2005, p. 1809

[27] F. Xiangning and S. Yulin, "Improvement on leach protocol of wireless sensor network," in 2007 International Conference on Sensor Technologies and Applications (SENSORCOMM 2007). IEEE, 2007, pp. 260264.

[28] M. B. Yassein, Y. Khamayseh, and W. Mardini, "Improvement on leach protocol of wireless sensor network (vleach," in Int. J. Digit. Content Technol. Appl. 2009. Citeseer, 2009.

[29] A. Al-Baz and A. El-Sayed, "A new algorithm for cluster head selection in leach protocol for wireless sensor networks," International journal of communication systems, vol. 31, no. 1, p. e3407, 2018.

[30] A. Rahmanian, H. Omranpour, M. Akbari, and K. Raahemifar, "A novel genetic algorithm in leach-c routing protocol for sensor networks," in 2011 24th Canadian Conference on Electrical and Computer Engineering (CCECE). IEEE, 2011, pp. 001 096-001 100.

[31] J.-L. Liu and C. V. Ravishankar, "Leach-ga: Genetic algorithm-based energy-efficient adaptive clustering protocol for wireless sensor networks," International Journal of Machine Learning and Computing, vol. 1, no. 1, p. 79, 2011.

[32] W. B. Heinzelman, A. P. Chandrakasan, H. Balakrishnan et al., "An application-specific protocol architecture for wireless microsensor networks," IEEE Transactions on wireless communications, vol. 1, no. 4, pp. 660-670, 2002.

[33] X. Li, L. Xu, H. Wang, J. Song, and S. X. Yang, "A differential evolution-based routing algorithm for environmental monitoring wireless sensor networks," Sensors, vol. 10, no. 6, pp. 5425-5442, 2010.

[34] D. Lin and Q. Wang, "A game theory based energy efficient clustering routing protocol for wsns," Wireless Networks, vol. 23, no. 4, pp. 11011111,2017

[35] Q. Wang, D. Lin, P. Yang, and Z. Zhang, "An energy-efficient compressive sensing-based clustering routing protocol for wsns," IEEE Sensors Journal, vol. 19, no. 10, pp. 3950-3960, 2019.

[36] A. M. Zungeru, L.-M. Ang, and K. P. Seng, "Classical and swarm intelligence based routing protocols for wireless sensor networks: A survey and comparison," Journal of Network and Computer Applications, vol. 35 , no. 5, pp. 1508-1536, 2012.
[37] P. K. Agarwal and C. M. Procopiuc, "Exact and approximation algorithms for clustering," Algorithmica, vol. 33, no. 2, pp. 201-226, 2002

[38] S. Guru, S. Halgamuge, and S. Fernando, "Particle swarm optimisers for cluster formation in wireless sensor networks," in 2005 International Conference on Intelligent Sensors, Sensor Networks and Information Processing. IEEE, 2005, pp. 319-324.

[39] B. Singh and D. K. Lobiyal, "A novel energy-aware cluster head selection based on particle swarm optimization for wireless sensor networks," Human-Centric Computing and Information Sciences, vol. 2, no. 1, p. 13, 2012.

[40] P. S. Rao and H. Banka, "Energy efficient clustering algorithms for wireless sensor networks: novel chemical reaction optimization approach," Wireless Networks, vol. 23, no. 2, pp. 433-452, 2017.

[41] P. Kuila, S. K. Gupta, and P. K. Jana, "A novel evolutionary approach for load balanced clustering problem for wireless sensor networks," Swarm and Evolutionary Computation, vol. 12, pp. 48-56, 2013.

[42] D. C. Hoang, P. Yadav, R. Kumar, and S. K. Panda, "Real-time implementation of a harmony search algorithm-based clustering protocol for energy-efficient wireless sensor networks," IEEE transactions on industrial informatics, vol. 10, no. 1, pp. 774-783, 2013.

[43] C. S. M. Cisse, K. Ahmed, C. Sarr, and M. A. Gregory, "Energy efficient hybrid clustering algorithm for wireless sensor network," in 2016 26th International Telecommunication Networks and Applications Conference (ITNAC). IEEE, 2016, pp. 38-43.

[44] S. Bhushan, R. Pal, and S. G. Antoshchuk, "Energy efficient clustering protocol for heterogeneous wireless sensor network: A hybrid approach using ga and k-means," in 2018 IEEE Second International Conference on Data Stream Mining \& Processing (DSMP). IEEE, 2018, pp. 381385.

[45] J. Xu, W. Liu, F. Lang, Y. Zhang, and C. Wang, "Distance measurement model based on rssi in wsn," Wireless Sensor Network, vol. 2, no. 08 , p. 606,2010

[46] J. Wang, Y. Cao, B. Li, H.-j. Kim, and S. Lee, "Particle swarm optimization based clustering algorithm with mobile sink for wsns," Future Generation Computer Systems, vol. 76, pp. 452-457, 2017. 